\title{
Perfusate adsorption during ex vivo lung perfusion improves early post-transplant lung function
}

Ilker Iskender, MD, MSc, ${ }^{\mathrm{a}}$ Stephan Arni, PhD, ${ }^{\mathrm{a}}$ Tatsuo Maeyashiki, MD, ${ }^{\mathrm{a}}$ Necati Citak, MD, ${ }^{\mathrm{a}}$ Mareike Sauer, DVM, ${ }^{\mathrm{b}}$ Josep Monné Rodriguez, DVM, ${ }^{\mathrm{c}}$ Thomas Frauenfelder, MD, ${ }^{\mathrm{d}}$ Isabelle Opitz, MD, ${ }^{\mathrm{a}}$ Walter Weder, MD, ${ }^{\mathrm{a}}$ and Ilhan Inci, $\mathrm{MD}^{\mathrm{a}}$

\section{ABSTRACT}

Objective: Improvement in ex vivo lung perfusion protocols could increase the number of donors available for transplantation and protect the lungs from primary graft dysfunction. We hypothesize that perfusate adsorption during ex vivo lung perfusion reconditions the allograft to ischemia-reperfusion injury after lung transplantation.

Methods: Donor pig lungs were preserved for 24 hours at $4^{\circ} \mathrm{C}$, followed by 6 hours of ex vivo lung perfusion according to the Toronto protocol. The perfusate was additionally adsorbed through a CytoSorb adsorber (CytoSorbents, Berlin, Germany) in the treatment group, whereas control lungs were perfused according to the standard protocol ( $\mathrm{n}=5$, each). Ex vivo lung perfusion physiology and biochemistry were monitored. Upon completion of ex vivo lung perfusion, a left single lung transplantation was performed. Oxygenation function and lung mechanics were assessed during a 4-hour reperfusion period. The inflammatory response was determined during ex vivo lung perfusion and reperfusion.

Results: The cytokine concentrations in the perfusate were markedly lower with the adsorber, resulting in improved ex vivo lung perfusion physiology and biochemistry during the 6-hour perfusion period. Post-transplant dynamic lung compliance was markedly better during the 4 -hour reperfusion period in the treatment group. Isolated allograft oxygenation function and dynamic compliance continued to be superior in the adsorber group at the end of reperfusion, accompanied by a markedly decreased local inflammatory response.

Conclusions: Implementation of an additional cytokine adsorber has refined the standard ex vivo lung perfusion protocol. Furthermore, cytokine removal during ex vivo lung perfusion improved immediate post-transplant graft function together with a less intense inflammatory response to reperfusion in pigs. Further studies are warranted to understand the beneficial effects of perfusate adsorption during ex vivo lung perfusion in the clinical setting. ( $J$ Thorac Cardiovasc Surg 2020; $\square$ :)

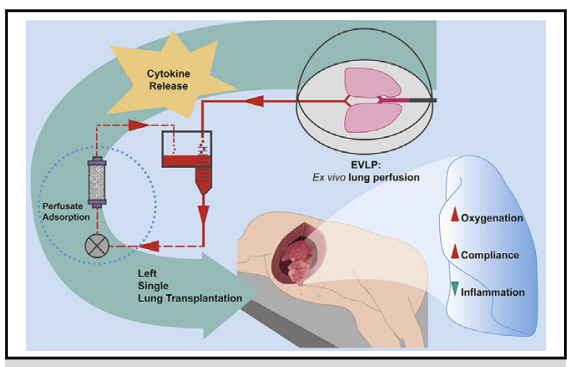

Perfusate adsorption during EVLP improves posttransplant lung function.

CENTRAL MESSAGE

Adsorption of inflammatory mediators during EVLP improves immediate post-transplant graft function by decreasing inflammatory response to reperfusion in pigs.

\section{PERSPECTIVE}

Current EVLP protocols are limited by short perfusion times and scarce treatment options. Advancements in the perfusion protocols may improve the quality of donor lungs and result in increased rates of use. Implementation of an additional broad-spectrum adsorber in perfusion circuits not only refines the current protocols but also improves short-term graft function.

See Commentary on page XXX.

\footnotetext{
From the Departments of ${ }^{\mathrm{a}}$ Thoracic Surgery and ${ }^{\mathrm{b}}$ Surgical Research, and ${ }^{\mathrm{d}}$ Institute of Diagnostic and Interventional Radiology, University Hospital Zurich-University of Zurich, Zurich, Switzerland; and ${ }^{\mathrm{c}}$ Department of Veterinary Pathology, University of Zurich, Zurich, Switzerland.

Funded by The CytoSorbents Europe GmbH

Read at the 99th Annual Meeting of The American Association for Thoracic Surgery, Toronto, Ontario, Canada, May 4-7, 2019.

Received for publication May 7, 2019; revisions received Nov 29, 2019; accepted for publication Dec 5, 2019.

Address for reprints: Ilhan Inci, MD, Department of Thoracic Surgery, University Hospital Zurich, Raemistrasse 100, Zurich 8091, Switzerland (E-mail: ilhan. inci@usz.ch).

$0022-5223 / \$ 36.00$

Copyright $\odot 2020$ by The American Association for Thoracic Surgery

https://doi.org/10.1016/j.jtcvs.2019.12.128
}

The implementation of ex vivo lung perfusion (EVLP) in donor lung management is the most significant advancement in lung transplantation over the last decade. ${ }^{1}$ EVLP initially was introduced as a second-look procedure to

Scanning this QR code will take
you to the article title page to
access supplementary informa-
tion. To view the AATS Annual
Meeting Webcast, see the URL
next to the webcast thumbnail.




$$
\begin{aligned}
& \text { Abbreviations and Acronyms } \\
& \begin{aligned}
\text { BWF } & =\text { bronchial wash fluid } \\
\text { EVLP } & =\text { ex vivo lung perfusion } \\
\text { IL } & =\text { interleukin } \\
\text { IL-1ra } & =\text { interleukin }-1 \text { receptor antagonist } \\
\text { IR } & =\text { ischemia-reperfusion } \\
\text { PGD } & =\text { primary graft dysfunction }
\end{aligned}
\end{aligned}
$$

high-risk donor lungs before transplantation. Growing experience with EVLP has ensured its safety and efficacy in lung transplantation. ${ }^{2}$ Centers across the world have successfully adopted the procedure and reported increased use of donor lungs. ${ }^{3,4}$

Other than donor lung assessment, EVLP also has a role in donor lung preservation. Accumulating evidence suggests that extension of donor ischemic time with an additional EVLP between the 2 cold static preservations does not have a negative impact on early outcomes of lung transplantation. ${ }^{5,6}$ Moreover, EVLP-driven preservation of the standard criteria donor lungs has been shown to be effective and safe regardless of the perfusion protocols. ${ }^{7,8}$

One of the limitations of the current EVLP protocols is the short perfusion time. To our knowledge, EVLP for more than 12 hours has not been reported so far using the 3 widely used EVLP protocols in the clinical setting. Several studies have been performed to optimize the EVLP protocols looking at the roles of atrial pressure, ${ }^{9}$ synchronous bronchial artery circulation, ${ }^{10}$ cellular and acellular perfusates, ${ }^{11}$ continued perfusate exchange, ${ }^{12}$ negative pressure ventilation, ${ }^{13}$ perfusate oxygen concentration, ${ }^{14}$ continuous perfusate adsorption, ${ }^{15}$ and positioning of donor lungs. ${ }^{16}$ A combination of these strategies may result in prolonged EVLP times.

EVLP simulates ischemia-reperfusion (IR) processes in lung transplantation, which is characterized by the release of inflammatory mediators into the perfusate. ${ }^{17}$ Kakishita and colleagues ${ }^{18}$ have shown the feasibility of cytokine removal with an adsorbent membrane during EVLP. Recently, we also demonstrated beneficial effects of perfusate adsorption during EVLP using a novel membrane (Cytosorb; CytoSorbents Inc, Monmouth Junction, NJ) in a prolonged cold ischemic injury model in pigs. ${ }^{15}$ In addition to marked cytokine removal, continuous perfusate adsorption improved the EVLP physiology and biochemistry in this setting. ${ }^{15}$ Moreover, increased expression of proinflammatory cytokines during EVLP has been shown to correlate with higher primary graft dysfunction (PGD) rates after lung transplantation. ${ }^{19}$ The present study is based on the hypothesis that perfusate adsorption during EVLP reconditions the allograft to IR-induced injury after lung transplantation and improves short-term graft function.
Improvement in EVLP could increase the number of donors available for transplantation and protect the lungs from PGD.

\section{MATERIALS AND METHODS}

All animals received humane care during experiments in accordance with the updated "The Guide for the Care and Use of Laboratory Animals" (8th Edition, US National Research Council). The animal use protocol was approved by the Veterinary Authorities of the Swiss Kanton of Zurich. Female domestic pigs were used.

\section{Pharmacokinetics of Meropenem and Methylprednisolone in Ex Vivo Lung Perfusion Perfusate From a Previous Trial}

The CytoSorb adsorber contains microporous beads, which are capable of adsorbing broad spectrum of mediators, including drugs ${ }^{20}{ }^{20}$ Thus, we first decided to analyze the perfusate samples collected at baseline, 1 hour, 3 hours, and in 3-hour intervals thereafter during our previous 12-hour EVLP trial. ${ }^{15}$ The 2 target drugs, methylprednisolone (Medrol, Pfizer, New York, NY) and meropenem (Meronem, Pfizer), were extracted from perfusate samples using a methanol precipitation. For pharmacokinetics, samples were analyzed by liquid chromatography-mass spectrometry using selected reaction-monitoring assays. The system consists of a Thermo TSQ-Quantiva coupled to a Waters M-class ultra-high performance liquid chromatography. The liquid chromatography was set up in reverse phase mode $(\mathrm{C}-18,150 \mu \mathrm{m} \times 5 \mathrm{~cm})$. External calibration curves, with a concentration range of 10 to $500 \mathrm{nM}$, were included. Quantification was performed using the Xcalibur Quan Browser software (Thermo Fisher Scientific, Waltham, Mass).

\section{Lung Retrieval, Ex Vivo Lung Perfusion, and Lung Transplantation Procedures}

The experimental procedures have been described in detail. ${ }^{21}$ The lungs were kept in a cold room for 24 hours at $4{ }^{\circ} \mathrm{C}$ after pneumoplegia with Perfadex (XVIVO Perfusion, Göteborg, Sweden). EVLP was performed according to the Toronto protocol for 6 hours. ${ }^{2}$ Perfusions were performed with or without the CytoSorb adsorber, commercially available for use in Europe with a CE mark (CytoSorbents Inc, Monmouth Junction, NJ) according to an established methodology in a randomized fashion $(\mathrm{n}=5$, each). ${ }^{15}$ Recipient operation was started at 5 hours of EVLP. After a thoracotomy, the right pulmonary artery was encircled followed by a left pneumonectomy. Orthotopic left single lung transplantation was then performed, and the recipients were observed for 4 hours.

\section{Physiologic Assessment During Ex Vivo Lung Perfusion and After Transplantation}

The allograft function was assessed hourly during EVLP and after reperfusion by means of pulmonary gas exchange, lung mechanics, and vascular pressures. At the end of 4 hours of reperfusion, the allograft was challenged with a contralateral pulmonary artery occlusion for 5 minutes, followed by clamping of the right main bronchus to a target pressure of $30 \mathrm{cmH}_{2} \mathrm{O}$ for evaluation of the isolated allograft mechanics.

\section{Perfusate, Plasma, Bronchial Wash Fluid, and Tissue Collection and Analyses}

Perfusate and plasma samples were collected hourly. The Epoc blood analysis system (Epocal, Inc, Ottawa, ON, Canada) was used to measure perfusate and blood gases and for biochemistry.

At the end of EVLP and transplantation, a bronchoalveolar wash was performed with $20 \mathrm{~mL}$ of cold saline from the lower lobe segments of the right and left lungs, respectively. Approximately $15 \mathrm{~mL}$ were recovered 


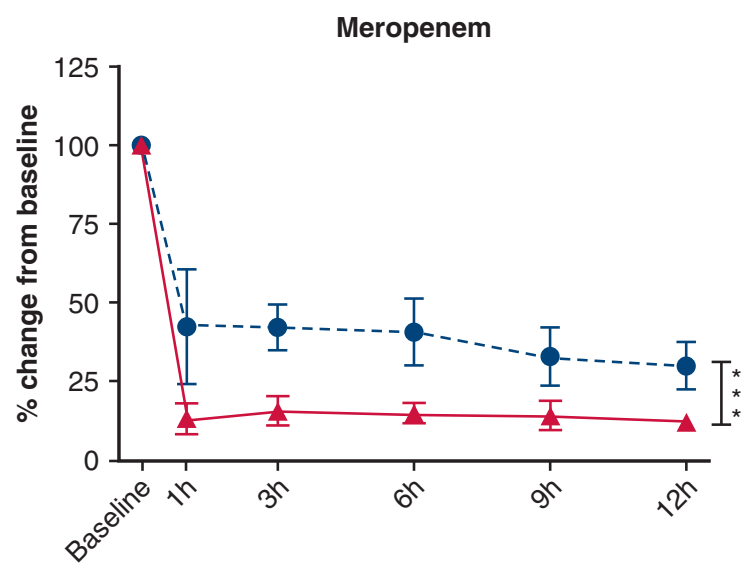

Time points

\section{A}

Control $\rightarrow$ Adsorber

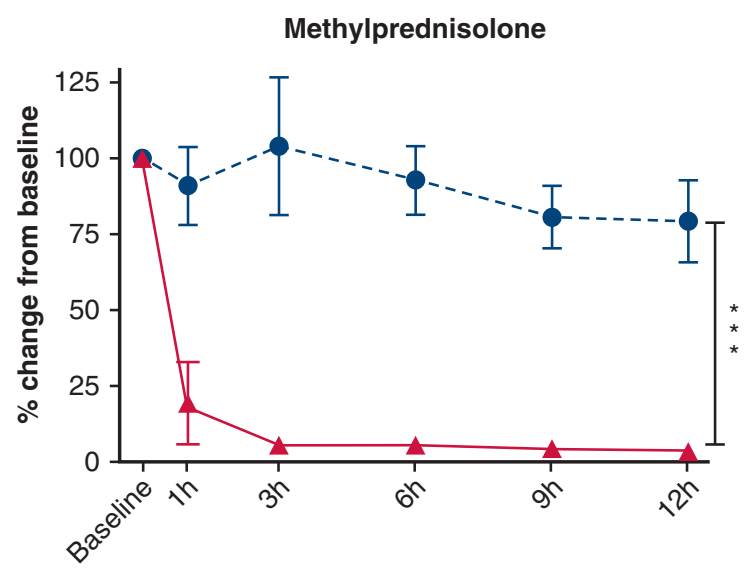

Time points

B

- Control $\rightarrow$ - Adsorber

FIGURE 1. Pharmacokinetics of meropenem and methylprednisolone during the 12-hour of EVLP period. Perfusate concentrations of meropenem (A) and methylprednisolone (B) were measured at baseline, 1 hour, 3 hours, and in 3-hour intervals thereafter in samples collected during the initial trial. ${ }^{15}$ The perfusate concentrations of meropenem and methylprednisolone were markedly decreased in the adsorber group compared with the control group (*** $P<.001$, between groups). The drug removal effect of the adsorber was more prominent for methylprednisolone than meropenem. Data are presented as percentage change from baseline and expressed as mean \pm standard deviation.

during each wash. The recovered wash fluid was subjected to cytologic assessment at the hospital's core laboratories, using May-GrünwaldGiemsa-stained cytologic specimens and subsequently processed as previously described for further analysis. ${ }^{22}$ Likewise, lung tissue samples were also collected at the end of each procedure.

\section{Assessment of the Inflammatory Response}

The inflammatory response was analyzed in perfusate, plasma, and bronchial wash fluid (BWF) samples using Milliplex MAP Porcine Cytokine/Chemokine Magnetic Bead Panel (EMD Millipore, Billerica, Mass) by a luminex laboratory at the Mouse Metabolic Evaluation Facility of the University of Lausanne (Switzerland). Additionally, tissue expressions of interleukin (IL)-1 $\beta$ and IL-8 were analyzed as previously described. ${ }^{23}$

\section{Protein Assay}

The Pierce micro BCA kit was used to measure the total protein concentrations in perfusate, BWF, tissue, and plasma samples according to the manufacturer's instructions (Thermo Scientific, Rockford, Ill).

\section{Radiologic Assessment of the Lungs}

Lung $\mathrm{X}$-rays taken at the end of EVLP were evaluated by a radiologist in a blinded fashion as previously described. ${ }^{15}$

\section{Histologic Assessment of the Lungs}

Tissue samples taken at the end of EVLP and transplantation were stained with hematoxylin-eosin and blindly assessed by 2 veterinary pathologists for any pathological changes. The most prominent features observed in the transplanted lungs were used to develop a scoring system. This was as follows: (A) evidence of neutrophil recruitment into the lungs, represented by the presence of neutrophils within vessels (1: moderate numbers, 2: high numbers, 3: vessels packed with neutrophils); (B) evidence of neutrophil emigration into the tissue (1: neutrophil rolling along vascular endothelial cells, 2 : neutrophils within vessel walls, 3 : neutrophils immediately outside vessels); and (C) evidence of neutrophils within the tissue, represented by the presence of individual neutrophils within alveolar lumina (1: in rare alveoli, 2: in occasional alveoli, 3: in numerous alveoli).

\section{Statistical Analyses}

All results are expressed as mean \pm standard deviation. Mann-Whitney $U$ test was used, where data were noncontinuous. Two-way analysis of variance for repeated measures was used, where such data contained a time component. Statistical analyses were performed with PRISM 5 software (GraphPad Software, Inc, La Jolla, Calif).

\section{RESULTS}

Effects of the Adsorber on Pharmacokinetics of Meropenem and Methylprednisolone Under Ex Vivo Lung Perfusion Conditions

Meropenem levels were diminished by more than $50 \%$ of the baseline at 1 hour and remained stable throughout perfusions in the control group (Figure 1, A). Methylprednisolone levels were sustained at approximately $80 \%$ of the baseline during the 12 hours of EVLP period in the control group (Figure 1, $B$ ). These results may indicate that tissue absorption of meropenem was higher compared with prednisolone during EVLP. However, the perfusate concentrations of meropenem and methylprednisolone were markedly decreased in the adsorber group compared with the control group $(P<.001$, Figure $1, A$ and $B)$. The drugremoval effect of the adsorber was more prominent for methylprednisolone than meropenem.

\section{Reproducibility of Ex Vivo Lung Perfusion Part}

Preretrieval characteristics of donor animals. Donor body weight (control: $29.8 \pm 3.6 \mathrm{~kg}$ vs adsorber: $28.4 \pm 1.5 \mathrm{~kg} ; P=.67$ ), donor static compliance (control: $23.6 \pm 2.5 \mathrm{~mL} / \mathrm{mbar}$ vs adsorber: $25.3 \pm 3.5 \mathrm{~mL} / \mathrm{mbar}$; $P=.42$ ), and cold ischemic time (control: $24.1 \pm 0.3$ hours vs adsorber: $24.3 \pm 0.3$ hours; $P=.42$ ) 


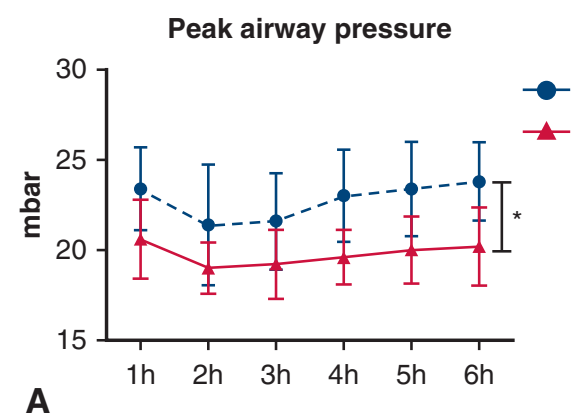

Delta $\mathrm{PaO}_{2}$

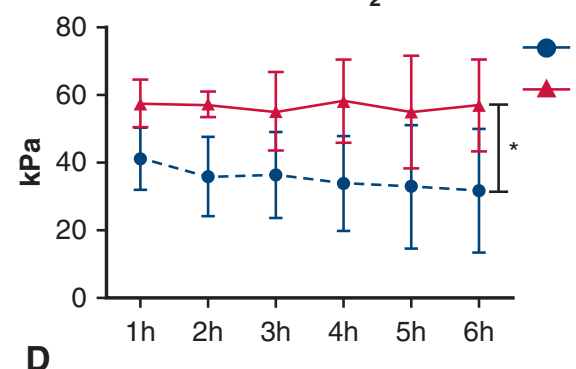

Calcium

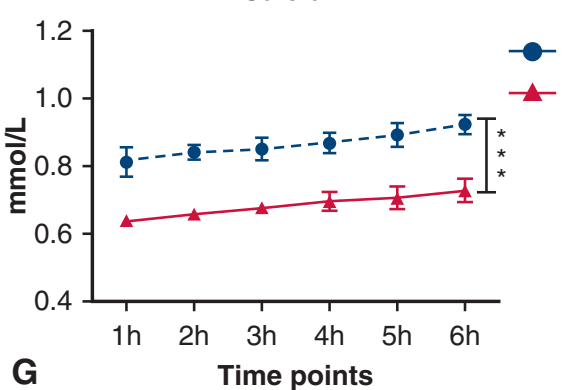

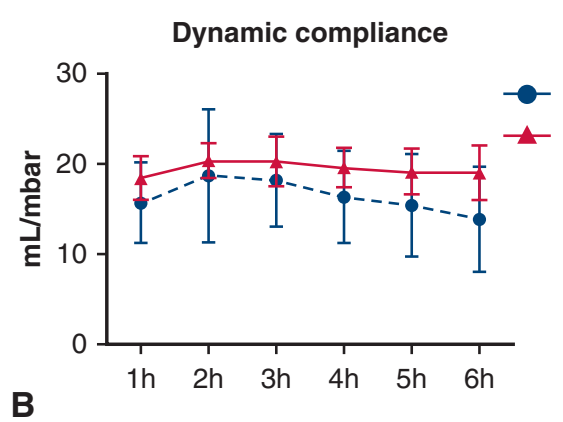

$\mathrm{pH}$

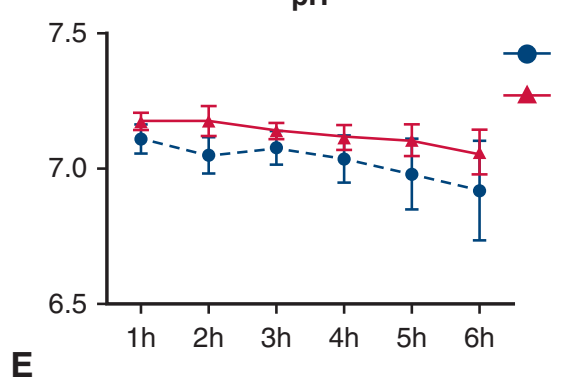

Glucose

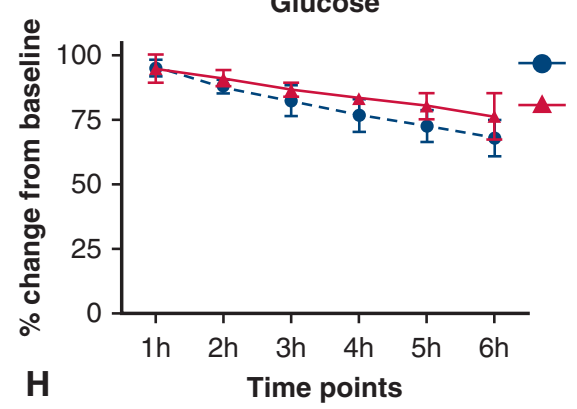

Pulmonary vascular resistance

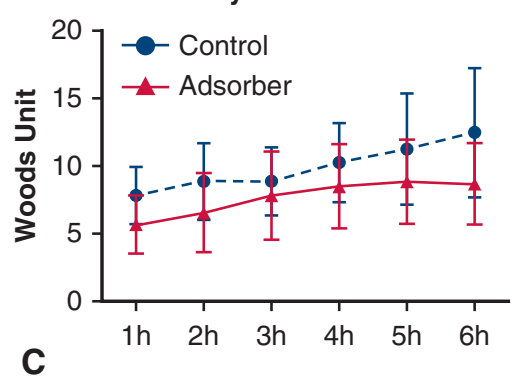

Potassium

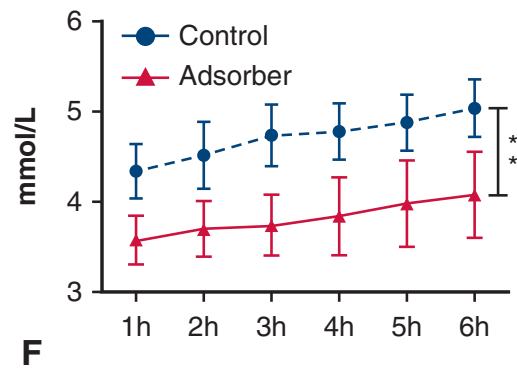

Lactate

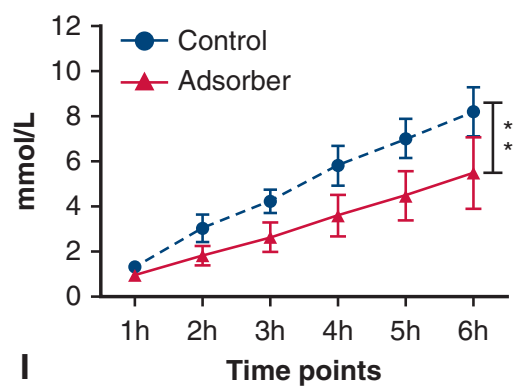

FIGURE 2. EVLP physiology and biochemistry. Pulmonary mechanics, vascular pressures, oxygenation function, and perfusate gas analyses were recorded hourly during the 6-hour EVLP period. EVLP physiology, determined by peak airway pressure (A) and delta $\mathrm{PO}_{2}$ (D), was significantly better in the treatment group. EVLP biochemistry, determined by potassium (F), calcium (G), and lactate (I) levels, was also improved in the adsorber group. Dynamic compliance (B), pulmonary vascular resistance $(\mathrm{C}), \mathrm{pH}(\mathrm{E})$, and glucose $(\mathrm{H})$ did not reach a statistical difference. Pulmonary vascular resistance $=$ pulmonary artery pressure - (left atrial pressure/flow). Delta $\mathrm{PO}_{2}$ is left atrial pressure of oxygen - pulmonary arterial pressure of oxygen. Data are expressed as mean \pm standard deviation $(* P<.05, * * P<.01, * * * P<.001$, between groups).

did not differ significantly between the 2 groups. Of note, the last partial pressure of arterial oxygen/fraction of inspired oxygen ratio was markedly higher in the control group compared with the adsorber group $(65.9 \pm 3.5 \mathrm{kPa}$ vs $57 \pm 4.8 \mathrm{kPa} ; P=.008)$.

Ex vivo lung perfusion physiology and biochemistry. During the 6-hour EVLP period, peak airway pressure was significantly lower in the adsorber group $(P=.045$, Figure $2, A)$. Dynamic compliance $(P=.27$, Figure $2, B)$ and pulmonary vascular resistance $(P=.26$, Figure $2, C)$ did not differ significantly between the 2 groups. Moreover, the pulmonary veno-arterial oxygen pressure gradient was markedly better in the adsorber group $(P=.016$, Figure $2, D)$.

Electrolytes, such as hydrogen, potassium, and calcium, continued to accumulate in the perfusate during the 6-hour EVLP period (Figure 2, $E-G$ ). However, a trend toward a more physiologic $\mathrm{pH}(P=.055$, Figure $2, E)$ and markedly decreased potassium $(P=.003$, Figure $2, F)$ and calcium $(P<.001$, Figure $2, G)$ levels was observed in the adsorber group.

Likewise, a gradual decrease in perfusate glucose concentrations combined with high lactate levels were the characteristics of pulmonary metabolism during EVLP (Figure 2, $H$ and $I$ ). There was a trend toward lower glucose consumption rates $(P=.059$, Figure $2, H)$ and a significant decrease in lactate production in the adsorber group compared with the controls $(P=.004$, Figure $2, I)$.

Inflammatory response. Perfusate samples collected at 3 hours and 6 hours of EVLP were analyzed using a multiplex kit. Overall, the perfusate levels of IL- $1 \alpha$, IL-1 $\beta$, IL-1 
receptor antagonist (IL-1ra), IL-6, IL-8, IL-10, IL-12, and IL-18 were found to gradually increased over time in the control group (Table 1). However, all analytes were significantly lower in the adsorber group during the 6-hour EVLP period. Furthermore, the perfusate total protein concentration was also markedly lower in the adsorber group $(80.2 \pm 6$ vs $64.5 \pm 8.3 \mathrm{mg} / \mathrm{mL} ; P=.032)$.

End ex vivo lung perfusion assessments. The cytologic examination found that macrophages were the predominant cell type in the BWF in both groups (control: $85.9 \% \pm 9.3 \%$ vs adsorber: $89.1 \% \pm 5.7 \% ; P=.68$ ). The following parameters did not differ significantly, but they were lower in the treatment group: perfusate consumption (control: $536 \pm 163 \mathrm{~mL}$ vs adsorber: $394 \pm 119 \mathrm{~mL}$; $P=.31$ ) and radiologic lung injury scores (control: $6.4 \pm 2$ vs adsorber: $4.4 \pm 1.5 ; P=.205$ ). In both groups, the histologic changes were comparable and restricted to mild to moderate subpleural and interstitial edema and the presence of apoptotic leukocytes within vessel lumina and, to a variable extent, within the occasionally observed mild bronchus associated lymphatic tissue (Figure E1, $A$ ).

\section{Outcomes of Lung Transplantation}

Baseline characteristics of recipient animals. Recipient body weight (control: $32.2 \pm 2.2 \mathrm{~kg}$ vs adsorber: $32 \pm 1.7 \mathrm{~kg} ; P=1$ ), recipient static compliance (control: $25.8 \pm 3.8 \mathrm{~mL} / \mathrm{mbar}$ vs adsorber: $25 \pm 2.6 \mathrm{~mL} / \mathrm{mbar}$; $P=.92)$, the partial pressure of arterial oxygen/fraction of inspired oxygen ratio before thoracotomy (control: $64.6 \pm 6 \mathrm{kPa}$ vs adsorber: $61.5 \pm 6 \mathrm{kPa} ; P=.69$ ), and warm ischemic time (control: $71.4 \pm 8.7$ minutes vs adsorber: $76 \pm 4.2$ minutes; $P=.45$ ) did not differ significantly between the 2 groups.

Post-transplant physiologic parameters. Recipient animals were supported hemodynamically using Ringer's lactate solution throughout the procedure. Overall, mean arterial pressures were comparable between the 2 groups during the experimental procedures $(P=.135$, Figure $3, A)$.

Dynamic pulmonary compliance was significantly higher throughout reperfusion in the adsorber group $(P=.031$, Figure $3, B)$. Although not reaching a statistical significance, systemic arterial $(P=.099$, Figure $3, C)$ and pulmonary venous $(P=.175$, Figure $3, D)$ oxygenation function were superior in the treatment group.

After clamping the right pulmonary artery, allograft continued to provide a superior gas exchange function in the treatment group compared with control $(37 \pm 17 \mathrm{kPa}$ vs $61.8 \pm 11.8 \mathrm{kPa} ; P=.056$, Figure $3, E$ ). Likewise, isolated dynamic compliance was significantly better in the adsorber group $(7.7 \pm 3.2 \mathrm{~mL} / \mathrm{mbar}$ vs $12.9 \pm 2.2 \mathrm{~mL} /$ mbar; $P=.032$, Figure $3, D$ ).

Post-transplant inflammatory response. The inflammatory response was assessed in plasma collected at baseline, 2 hours, and 4 hours of reperfusion, and BWF samples were collected at autopsy for 8 analytes. The plasma levels of IL$1 \mathrm{ra}$, an acute phase protein, were significantly lower in the absorber group during the 4-hour reperfusion period $(P=.009$, Figure 4$)$. Other analytes in the plasma did not differ significantly between the 2 groups, and the results are not presented. When we looked at the BWF samples, except for IL- $1 \alpha$, measurements of all other analytes were lower in the treated lungs (Table 2). For IL-1ra, IL-6, and

TABLE 1. Expression of cytokines and chemokines in perfusate at 3 and 6 hours of ex vivo lung perfusion

\begin{tabular}{lccc}
\hline & & Cytokine/chemokine levels in perfusate & \\
\hline Analytes & Time points & Control $(\mathbf{p g} / \mathbf{m L})$ & Adsorber $(\mathbf{p g} / \mathbf{m L})$ \\
\hline IL-1 $\alpha$ & $3 \mathrm{~h}$ & $54 \pm 49$ & $6 \pm 7$ \\
& $6 \mathrm{~h}$ & $67 \pm 50$ & $1 \pm 2$ \\
IL-1 $\beta$ & $3 \mathrm{~h}$ & $155 \pm 60$ & $51 \pm 24$ \\
& $6 \mathrm{~h}$ & $613 \pm 540$ & $81 \pm 45$ \\
IL-1ra & $3 \mathrm{~h}$ & $294 \pm 101$ & $63 \pm 32$ \\
& $6 \mathrm{~h}$ & $1214 \pm 535$ & $35 \pm 17$ \\
IL-6 & $3 \mathrm{~h}$ & $719 \pm 340$ & $602 \pm 352$ \\
& $6 \mathrm{~h}$ & $2833 \pm 821$ & $791 \pm 354$ \\
IL-8 & $3 \mathrm{~h}$ & $17,121 \pm 8944$ & $943 \pm 766$ \\
& $6 \mathrm{~h}$ & $28,812 \pm 11,105$ & $444 \pm 343$ \\
IL-10 & $3 \mathrm{~h}$ & $72 \pm 30$ & $24 \pm 18$ \\
& $6 \mathrm{~h}$ & $191 \pm 107$ & $4 \pm 3$ \\
IL-12 & $3 \mathrm{~h}$ & $185 \pm 63$ & $74 \pm 46$ \\
& $6 \mathrm{~h}$ & $404 \pm 120$ & $97 \pm 51$ \\
IL-18 & $3 \mathrm{~h}$ & $8068 \pm 4416$ & $245 \pm 94$ \\
& $6 \mathrm{~h}$ & $7980 \pm 3647$ & $131 \pm 112$ \\
\hline
\end{tabular}

Data are expressed as mean \pm standard deviation. Comparisons between the groups were made by 2-way repeated-measures analysis of variance. $I L$, Interleukin; $I L$ - $1 \mathrm{ra}$, IL-1 receptor antagonist. $* P<.05, * * P<.01,{ }^{* * *} P<.001$, between groups. 

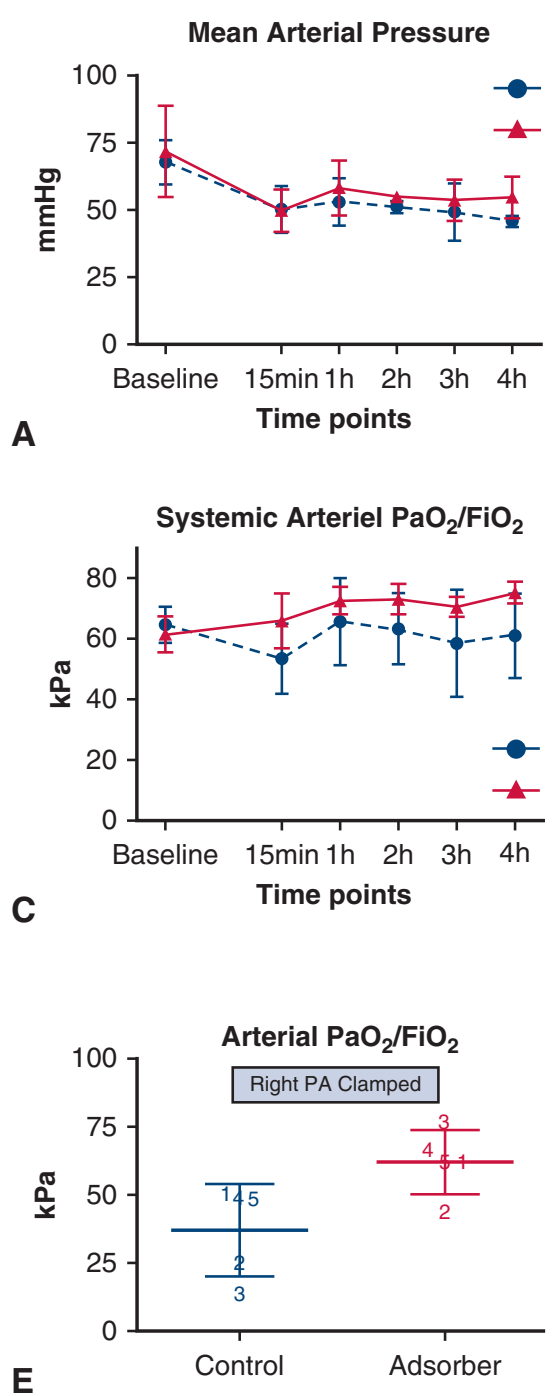

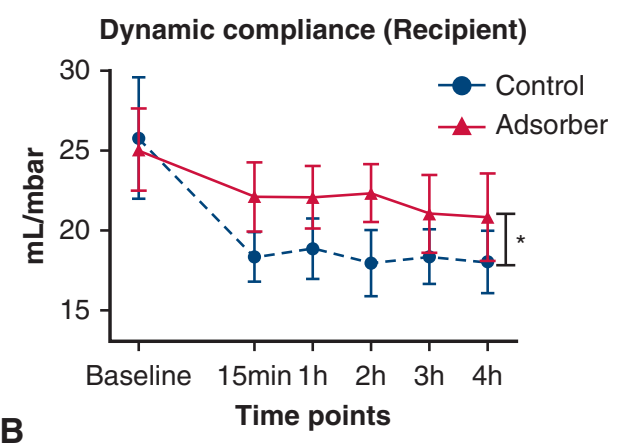

Pulmonary Vein $\mathrm{PaO}_{2} / \mathrm{FiO}_{2}$

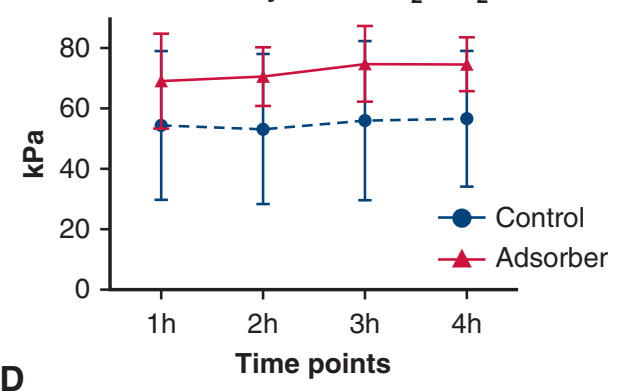

D

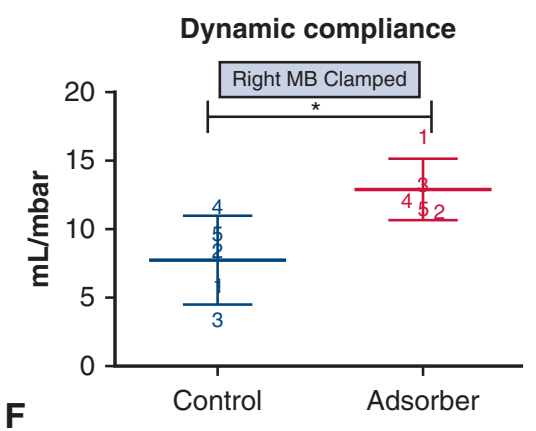

FIGURE 3. Post-transplant physiologic outcomes. Mean arterial pressure (A), dynamic compliance (B), and systemic arterial partial pressure of arterial oxygen/fraction of inspired oxygen ratio (C) were recorded at baseline, early after reperfusion, and hourly thereafter. Dynamic pulmonary compliance was significantly higher throughout reperfusion in the adsorber group $(\mathrm{B}, * P<.05$, between groups). Systemic arterial $(\mathrm{C}, P=.1)$ and pulmonary venous $(\mathrm{D}$, $P=.18$ ) oxygenation function were also better in the treatment group. After occlusion of the contralateral anatomic structures, isolated allograft oxygenation function $(\mathrm{E}, P=.056)$ and dynamic compliance $(\mathrm{F}, * P<.05$, between groups) continued to be better in the treatment group. Matching results for each animal are presented. $\mathrm{PaO}_{2} / \mathrm{FIO}_{2}$, Partial pressure of arterial oxygen/fraction of inspired oxygen; $\mathrm{PA}$, pulmonary artery; $\mathrm{MB}$, main bronchus. Data are expressed as mean \pm standard deviation.

IL-8, the difference was statistically significantly. In parallel with the BWF, tissue expressions of IL-1 $\beta$ (control: $4.4 \pm 0.8 \mathrm{ng} / \mathrm{mL}$ vs adsorber: $3.5 \pm 0.6 \mathrm{ng} / \mathrm{mL} ; P=.056$ ) and IL-8 (control: $213 \pm 125 \mathrm{pg} / \mathrm{mL}$ vs adsorber: $137 \pm 76 \mathrm{pg} / \mathrm{mL} ; P=.31$ ) were lower in the treatment group (Figure 5, upper panel). On the other hand, total protein concentration of plasma (control: $54.9 \pm 6.6 \mathrm{mg} / \mathrm{mL}$ vs adsorber: $52.9 \pm 3.4 \mathrm{mg} / \mathrm{mL} ; P=.84$ ), BWF samples (control: $62.9 \pm 52.4 \mathrm{mg} / \mathrm{mL}$ vs adsorber: $33.8 \pm 42.4 \mathrm{mg} / \mathrm{mL}$; $P=.22$ ), and tissue (control: $3.2 \pm 0.1 \mathrm{mg} / \mathrm{mL}$ vs adsorber: $3 \pm 0.3 \mathrm{mg} / \mathrm{mL} ; P=.31$ ) were comparable between the 2 groups.
Post-transplant assessments. BWF cytology at autopsy showed predominantly neutrophilic response in both groups (control: $59.3 \% \pm 23.9 \%$ vs adsorber: $44.2 \% \pm 22.4 \%$; $P=.42$ ). The histologic assessment of the lungs collected during the autopsy showed consistent changes in response to reperfusion, reflecting the recruitment of neutrophils into the lung. All animals exhibited moderate or high numbers of neutrophils in vascular lumina. In 1 animal in the control group, several vessels were packed with neutrophils (animal number 5, Figure 5,C). Vascular endothelial cells were generally found to be activated (Figure E1, B); this coincided with evidence of neutrophil rolling along 


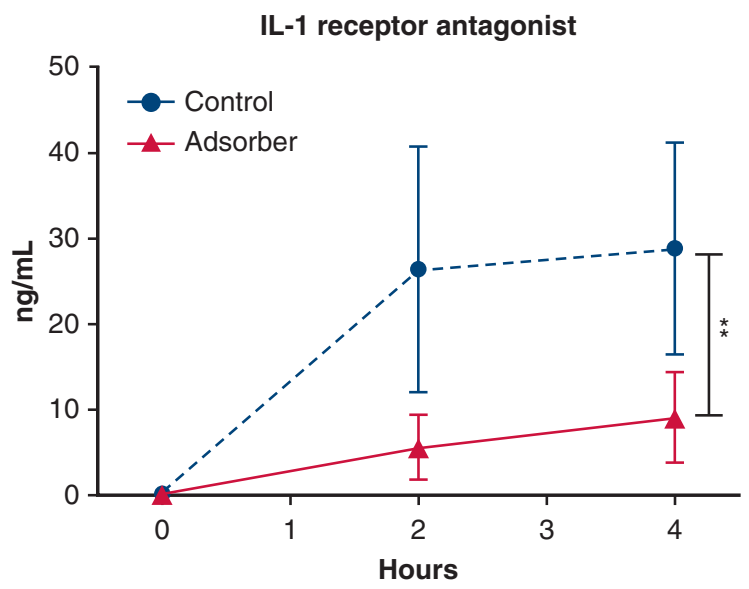

FIGURE 4. Plasma levels of soluble IL-1ra were significantly lower in the treatment group after reperfusion. Data are expressed as mean \pm standard deviation $(* * P<.01$, between groups). $I L$, Interleukin.

the endothelial cells, and in several animals also with proof of neutrophil emigration (Figure E1, C), represented by the presence of neutrophils in arterial walls and even in the perivascular tissue. In most lungs, neutrophils were found in alveoli (Figure E1, D). The overall microscopic lung injury scoring was comparable between the 2 groups $(5.2 \pm 2.7$ vs $4.2 \pm 1.6 ; P=1$, Figure $5, C$ ). However, the presence of individual neutrophils within alveolar lumina $(1.9 \pm 1$ vs $1 \pm 1 ; P=.29$, Figure $5, D$ ) was lower, indicating a less intense inflammatory response in the adsorber group. Furthermore, the discordance between the 2 pathologists related to the microscopic lung injury are shown in detail in Table E1.

\section{DISCUSSION}

In the present study, we have further tested the beneficial effects of perfusate adsorption during EVLP on short-term post-transplant lung function in a pig EVLP lung transplant model. With this strategy, we have repeatedly shown that
EVLP physiology and biochemistry were significantly improved. ${ }^{15}$ Furthermore, cytokine filtration during EVLP resulted in the preservation of post-transplant graft function, represented by enhanced gas exchange and lung mechanics, and a less intense inflammatory response to IR injury in pigs (Figure 6). We conclude that implementation of an additional cytokine adsorber has refined the standard EVLP protocol and may be beneficial to protect donor lungs from unwanted effects of PGD in lung transplantation.

Other than cytokines, the adsorber removes a wide range of molecules such as damage- and pathogen-associated molecular patterns, metabolites, hormones, proteins, and drugs. ${ }^{20}$ We have previously shown a certain albumin removal effect of the adsorber under EVLP conditions. ${ }^{15}$ Antibiotics, steroids, and heparin are generally added to the prime solution in EVLP protocols. Thus, we first determined the pharmacokinetics of meropenem and methylprednisolone in perfusate samples collected during our previous trial. The impact of the adsorber on drug removal from perfusate was remarkable for both meropenem and methylprednisolone, but the latter more prominently in this study. Of note, tissue adsorption of meropenem compared with methylprednisolone was higher in the control lungs. To our knowledge, the impacts of the empiric use of antibiotics and steroids in EVLP have not been studied; thus, the clinical relevance of the reduced levels of these substances remains unclear. Therefore, we have decided not to change the current regimen in the present study. However, dose adjustments are advised to reach therapeutic drug levels during treatment with CytoSorb in the EVLP setting.

Reproducibility in animal research and translation from bench to bedside are continuing to challenge the scientific community. Several guidelines have been published to improve reproducibility in research, yet the practice remains largely unchanged. ${ }^{24}$ We have previously shown the beneficial effects of continued perfusate adsorption with CytoSorb during a 12-hour EVLP period in pigs in a

TABLE 2. Inflammatory response in bronchial wash fluid samples at autopsy

\begin{tabular}{|c|c|c|c|}
\hline \multicolumn{4}{|c|}{ Cytokine/chemokine levels in bronchial wash fluid } \\
\hline Analytes & Control (pg/mL) & Adsorber (pg/mL) & $P$ value \\
\hline IL- $1 \alpha$ & $16 \pm 11$ & $16 \pm 16$ & 691 \\
\hline IL- $1 \beta$ & $358 \pm 305$ & $70 \pm 157$ & .056 \\
\hline IL-1 ra & $2411 \pm 2200$ & $218 \pm 133$ & $* * .008$ \\
\hline IL-6 & $898 \pm 207$ & $286 \pm 255$ & $* .016$ \\
\hline IL-8 & $1415 \pm 990$ & $301 \pm 259$ & $* .032$ \\
\hline IL-10 & $16 \pm 12$ & $6 \pm 7$ & .151 \\
\hline IL-12 & $125 \pm 73$ & $56 \pm 81$ & .095 \\
\hline IL-18 & $122 \pm 124$ & $9 \pm 13$ & .067 \\
\hline
\end{tabular}

Data are expressed as mean \pm standard deviation. Comparisons between the groups were made by the Mann-Whitney test. $I L$, Interleukin; IL-1 ra, IL-1 receptor antagonist. $* P<.05, * * P<.01$, between groups. 
Tissue IL-1 $\beta$

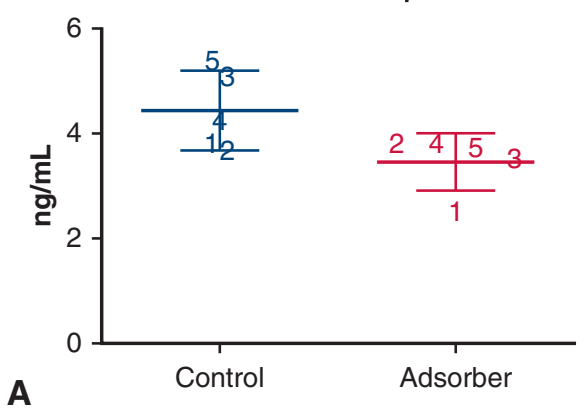

Microscopic lung injury

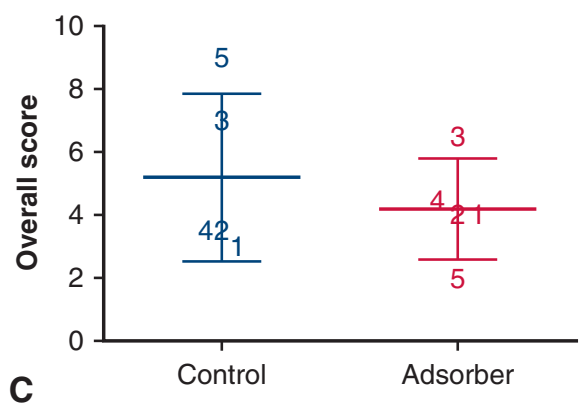

Tissue IL-8

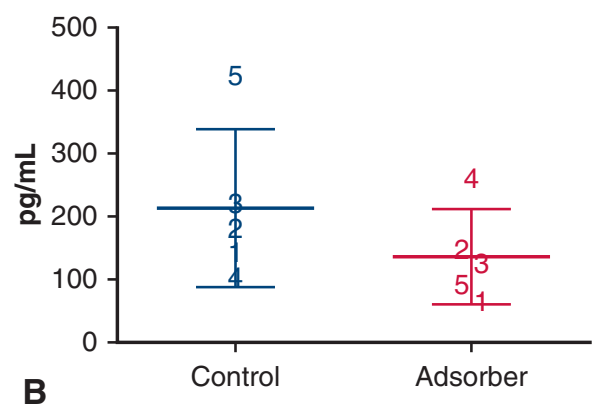

Precense of neutrophils within alveoli

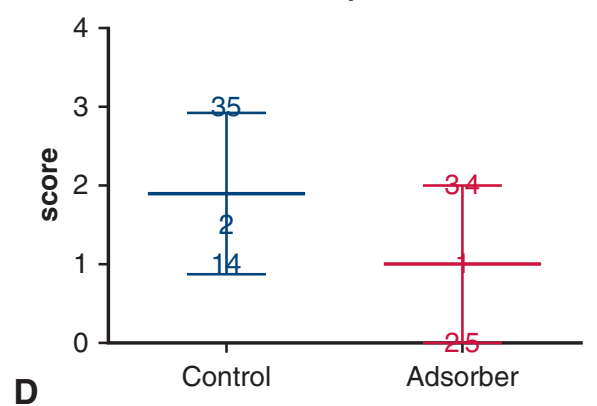

FIGURE 5. Correlation between tissue cytokine expression and histologic assessment of the allografts. Matching results for each animal are presented for tissue cytokines, IL-1 $\beta$ and IL-8 (upper panel), and microscopic lung injury, which was scored by 2 veterinary pathologists in a blinded fashion (lower panel). Tissue expressions of IL-1 $\beta(\mathrm{A}, P=.056)$ and IL-8 $(\mathrm{B}, P=.31)$ were lower in the treatment group. The overall microscopic lung injury scoring was comparable between the 2 groups $(\mathrm{C}, P=1)$. However, the presence of individual neutrophils within alveolar lumina $(\mathrm{D}, P=.29)$ was lower, indicating a less intense inflammatory response in the adsorber group. Data are expressed as mean \pm standard deviation. Comparison was made by Mann-Whitney $U$ test. $I L$, Interleukin.

proof-of-concept study. ${ }^{15}$ In the present study, we have decided to perfuse the lungs for 6 hours only to mimic the clinical scenario. Furthermore, at this time point, significant cytokine clearance was achieved, accompanied by improved EVLP physiology during the initial trial. ${ }^{15}$ Overall, the previous outcomes of the EVLP part are reproduced in the present study. The treated lungs exhibited less intense pulmonary edema, determined by decreased airway pressures, during the EVLP period; lesser pulmonary compliance and radiologic lung injury scores provide further evidence of decreased lung edema. The short perfusion time may have prevented these outcomes reaching a statistical significance and subsequent histologic evidence of quantitative differences. Oxygenation function of the treated lungs during EVLP was markedly better in the present study. Yet, gas exchange function during EVLP has been suggested as the least important parameter for assessing graft quality in acellular perfusion method. ${ }^{25}$ Other than physiologic parameters, perfusate analyses provide insights regarding the quality of donor lungs. Glucose consumption and lactate production have been suggested as predictive markers for decision making during EVLP. ${ }^{26}$ In parallel with our previous findings, perfusate adsorption led to markedly improved pulmonary metabolism in this study.
In combination with physiologic and biochemical parameters, novel biomarkers are needed to accurately predict post-transplant outcomes of EVLP-assessed donor lungs.

Accumulating evidence suggests that EVLP simulates IR injury in lung transplantation. Restoration of pneumocyte function under normothermic conditions induces enhanced cytokine expression during EVLP, ${ }^{17}$ which is similar to the inflammatory response that is seen after reperfusion with the recipients' blood in lung transplantation. ${ }^{27}$ Furthermore, an enhanced inflammatory response during EVLP has been shown to correlate with poor outcomes related to PGD. ${ }^{19}$ In line with our previous findings, the adsorber safely and effectively cleared the cytokines from the perfusate, resulting in improved EVLP physiology in this study. Kakishita and colleagues ${ }^{18}$ also showed the feasibility of cytokine removal during EVLP, yet the EVLP physiology did not improve under the experimental conditions they used. The adsorber device used in their study is composed of a cellulosic bead modified with a hexadecyl ligand. It is indicated for selective elimination of $\beta 2$-microglobulin from the circulating blood of patients with dialysis-related amyloidosis. ${ }^{28}$ This differs from CytoSorb, in which there are no ligands attached to the polymer, but which functions on size selectivity and surface adsorption. Furthermore, the 


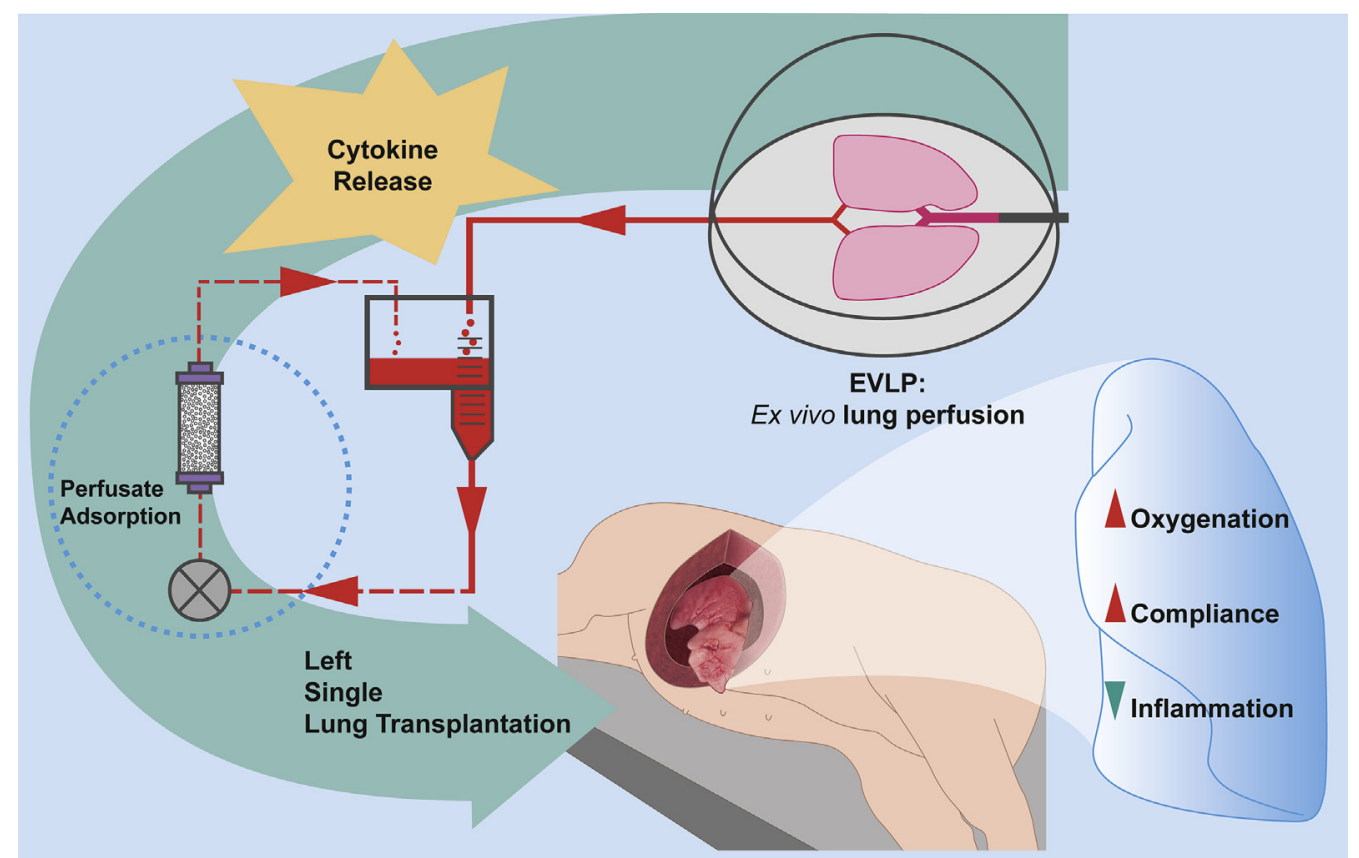

FIGURE 6. EVLP is characterized by the release of inflammatory mediators into the perfusate. In the present study, we have tested the beneficial effects of perfusate adsorption during EVLP on short-term post-transplant lung function in a pig EVLP lung transplant model. This strategy resulted in preservation of post-transplant graft function, by means of enhanced gas exchange and lung mechanics, via inhibition of inflammatory response to IR injury in pigs. $E V L P$, Ex vivo lung perfusion.

experimental conditions of the 2 studies were very different. The EVLP was initiated after a short warm ischemic time, whereas the present study used a clinically relevant prolonged cold ischemic injury model. ${ }^{29}$ However, donor lungs undergo different types of insults, such as brain death, aspiration, pneumonia, and barotrauma in the clinical setting. ${ }^{30}$ The broad spectrum adsorber used in this study has a capability to adsorb not only cytokines but also a wide range of other molecules. ${ }^{20}$ Thus, it is important to check the effects of perfusate adsorption in the clinical EVLP setting.

Donor lung reconditioning via perfusate adsorption during EVLP has led to a superior post-transplant lung function and decreased inflammatory response in this study. Donordriven alveolar macrophages have been thought to be the initiator of acute lung injury early after reperfusion. Cytokine release from alveolar macrophages further aggravates IR injury by the recruitment of recipient driven white blood cells into the allograft during the second phase of reperfusion. ${ }^{31}$ Expectedly, macrophages are the predominant cell type in BWF after EVLP, whereas a neutrophilic response became evident after reperfusion in this study. We further analyzed the local inflammatory response using a multiplex cytokine assay. For most analyzed cytokines/chemokines, levels were lower in the treated lungs, with IL-1ra, IL-6, and IL- 8 being markedly reduced. The inhibition of an inflammatory response during EVLP and after reperfusion is probably the mechanism underlying the protection against IR-related injury. Other than IL-1ra, the systemic inflammatory response to reperfusion did not differ significantly in this study. Likewise, the histologic examination showed that the type of inflammatory response (recruitment of neutrophils into the lungs) did not differ between treated and untreated lungs post-transplantation, whereas the extent of recruitment (with evidence of emigration and accumulation in alveolar lumina) was overall higher in untreated control lungs. IL- $1 \alpha$, IL- $1 \beta$, IL-1ra, and IL-18 belong to the IL-1 family cytokines that are being associated with acute and chronic inflammation. ${ }^{32}$ Although the recombinant form of IL-1ra has been used as an antiinflammatory drug in various inflammatory conditions, the soluble form is secreted by hepatocytes as an acute phase protein. ${ }^{33}$ We believe that elevated IL-1ra plasma levels are a surrogate marker of enhanced inflammation regulated by proinflammatory cytokines. Apparently, the main mechanism that underlies our results with Cytosorb is an increased protection against injury related to adsorption of inflammatory mediators. Nonetheless, we could not rule out that the mechanism behind this observed injury protection may be different, improving lung compliance or redirecting the perfusate flow to better oxygenated area.

\section{Study Limitations}

The main limitation of the present study is the lack of understanding the pathways involved in lung recovery during EVLP. Further studies are needed to understand the effects of the broad-spectrum adsorber on the mechanisms 
underlying IR injury in lung transplantation. We have conducted the study with the least possible number of animals to reach a conclusion. The duration of reperfusion was only 4 hours, which is far shorter than the clinical definition of PGD. The nonselective nature of the adsorber should be carefully considered when planning treatments with antiinflammatory molecules, such as IL-10. Taking into consideration of the limitations of the CytoSorb adsorber, modified EVLP protocol warrants further studies in the clinical setting.

\section{CONCLUSIONS}

Implementation of an additional cytokine adsorber has refined the standard EVLP protocol. Furthermore, cytokine removal during EVLP immediately improved posttransplant graft function and was associated with a less intense inflammatory response to reperfusion in pigs. Further studies are warranted to understand the beneficial effects of perfusate adsorption during EVLP in the clinical setting.

\section{Webcast}

You can watch a Webcast of this AATS meeting presentation by going to: https://aats.blob.core.windows.net/ media/19\%20AM/Saturday_May4/203AC/203AC/S34\% 20-\%20Lung \%20transplant \%20Donor/S34_4_webcast_ $022329348 . \mathrm{mp} 4$.

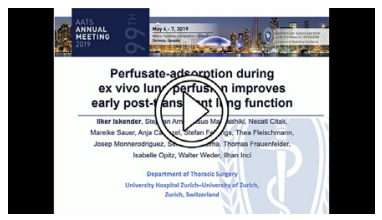

\section{Conflict of Interest Statement}

Dr Inci received research funds and Dr Iskender received speaker fees from the CytoSorbents Europe $\mathrm{GmbH}$. Dr Citak was supported with a fellowship grant from the Scientific and Technological Research Council of Turkey, Turkey. The study was partially funded by the CytoSorbents Europe GmbH, Germany, including provision of the CytoSorb cartridges used for the experiments. The research fund was used to cover the costs related to animal experimentation and bench works. None of the authors has received remuneration related to the work they provided in this study. The authors designed the study and responsible for data collection, interpretation of data, writing the manuscript, and the decision to submit for publication. The funding source had no role in the study design, data collection, interpretation of the data, preparation of the manuscript, or the decision to publish. All other authors have nothing to disclose with regard to commercial support.
The authors thank Anja Kipar, Laboratory of Animal Model Pathology, Vetsuisse Faculty, University of Zurich, during the revision of the article; and Anja Caviezel, Stefan Fehlings, Thea Fleischmann, and Serena Di Palma for their valuable contribution to this study.

\section{References}

1. Cypel M, Yeung JC, Liu M, Anraku M, Chen F, Karolak W, et al. Normothermic ex vivo lung perfusion in clinical lung transplantation. N Engl J Med. 2011;364: 1431-40.

2. Cypel M, Yeung JC, Machuca T, Chen M, Singer LG, Yasufuku K, et al. Experience with the first 50 ex vivo lung perfusions in clinical transplantation. $J$ Thorac Cardiovasc Surg. 2012;144:1200-6.

3. Sage E, Mussot S, Trebbia G, Puyo P, Stern M, Dartevelle P, et al. Lung transplantation from initially rejected donors after ex vivo lung reconditioning: the French experience. Eur J Cardiothorac Surg. 2014;46:794-9.

4. Nilsson T, Wallinder A, Henriksen I, Nilsson JC, Ricksten SE, MollerSorensen $\mathrm{H}$, et al. Lung transplantation after ex vivo lung perfusion in two Scandinavian centres. Eur J Cardiothorac Surg. 2019;55:766-72.

5. Yeung JC, Krueger T, Yasufuku K, de Perrot M, Pierre AF, Waddell TK, et al. Outcomes after transplantation of lungs preserved for more than $12 \mathrm{~h}$ : a retrospective study. Lancet Respir Med. 2017;5:119-24.

6. Ceulemans LJ, Monbaliu D, Verslype C, van der Merwe S, Laleman W, Vos R, et al. Combined liver and lung transplantation with extended normothermic lung preservation in a patient with end-stage emphysema complicated by druginduced acute liver failure. Am J Transplant. 2014;14:2412-6.

7. Warnecke G, Van Raemdonck D, Smith MA, Massard G, Kukreja J, Rea F, et al. Normothermic ex-vivo preservation with the portable organ care system lung device for bilateral lung transplantation (INSPIRE): a randomised, open-label, noninferiority, phase 3 study. Lancet Respir Med. 2018;6:357-67.

8. Slama A, Schillab L, Barta M, Benedek A, Mitterbauer A, Hoetzenecker K, et al. Standard donor lung procurement with normothermic ex vivo lung perfusion: a prospective randomized clinical trial. J Heart Lung Transplant. 2017:36:744-53.

9. Linacre V, Cypel M, Machuca T, Nakajima D, Hashimoto K, Zamel R, et al. Importance of left atrial pressure during ex vivo lung perfusion. J Heart Lung Transplant. 2016;35:808-14.

10. Tanaka Y, Noda K, Isse K, Tobita K, Maniwa Y, Bhama JK, et al. A novel dual ex vivo lung perfusion technique improves immediate outcomes in an experimental model of lung transplantation. Am J Transplant. 2015;15:1219-30.

11. Loor G, Howard BT, Spratt JR, Mattison LM, Panoskaltsis-Mortari A, Brown RZ, et al. Prolonged EVLP using OCS lung: cellular and acellular perfusates. Transplantation. 2017;101:2303-11.

12. Cheung HY. Strategies to Improve and Stabilize Extended Ex Vivo Lung Perfu sion. Master of Health Science Thesis. Toronto: University of Toronto; 2017.

13. Aboelnazar NS, Himmat S, Hatami S, White CW, Burhani MS, Dromparis P, et al. Negative pressure ventilation decreases inflammation and lung edema during normothermic ex-vivo lung perfusion. J Heart Lung Transplant. 2018;37: 520-30.

14. Noda K, Tane S, Haam SJ, Hayanga AJ, D’Cunha J, Luketich JD, et al. Optimal ex vivo lung perfusion techniques with oxygenated perfusate. J Heart Lung Transplant. 2017;36:466-74.

15. Iskender I, Cosgun T, Arni S, Trinkwitz M, Fehlings S, Yamada Y, et al. Cytokine filtration modulates pulmonary metabolism and edema formation during ex vivo lung perfusion. J Heart Lung Transplant. 2018;37:283-91.

16. Ordies S, Frick AE, Claes S, Schols D, Verleden SE, Van Raemdonck DE, et al. Prone positioning during ex vivo lung perfusion influences regional edema accumulation. J Surg Res. 2019;239:300-8.

17. Sadaria MR, Smith PD, Fullerton DA, Justison GA, Lee JH, Puskas F, et al. Cytokine expression profile in human lungs undergoing normothermic ex-vivo lung perfusion. Ann Thorac Surg. 2011;92:478-84.

18. Kakishita T, Oto T, Hori S, Miyoshi K, Otani S, Yamamoto S, et al. Suppression of inflammatory cytokines during ex vivo lung perfusion with an adsorbent membrane. Ann Thorac Surg. 2010;89:1773-9.

19. Machuca TN, Cypel M, Yeung JC, Bonato R, Zamel R, Chen M, et al. Protein expression profiling predicts graft performance in clinical ex vivo lung perfusion. Ann Surg. 2015;261:591-7.

20. Poli EC, Rimmelé T, Schneider AG. Hemoadsorption with CytoSorb ${ }^{\circledR}$. Intensive Care Med. 2019;45:236-9. 
21. Cosgun T, Iskender I, Yamada Y, Arni S, Lipiski M, van Tilburg K, et al. Ex vivo administration of trimetazidine improves post-transplant lung function in pig model. Eur J Cardiothorac Surg. 2017;52:171-7.

22. Inci I, Ampollini L, Arni S, Jungraithmayr W, Inci D, Hillinger S, et al. Ex vivo reconditioning of marginal donor lungs injured by acid aspiration. $J$ Heart Lung Transplant. 2008:27:1229-36.

23. Yamada Y, Iskender I, Arni S, Hillinger S, Cosgun T, Yu K, et al. Ex vivo treatment with inhaled $\mathrm{N}$-acetylcysteine in porcine lung transplantation. J Surg Res. 2017;218:341-7.

24. Smith AJ, Clutton RE, Lilley E, Hansen KEA, Brattelid T. PREPARE: guidelines for planning animal research and testing. Lab Anim. 2018;52:135-41.

25. Yeung JC, Cypel M, Machuca TN, Koike T, Cook DJ, Bonato R, et al. Physiologic assessment of the ex vivo donor lung for transplantation. J Heart Lung Transplant. 2012;31:1120-6.

26. Slama A, Barta M, Schillab L, Mitterbauer A, Jaksch P, Hoetzenecker K, et al. Metabolic assessment of marginal donor lungs during ex-vivo perfusion (EVLP): new parameters for decision making. J Heart Lung Transplant. 2015; 34(Suppl):S97.

27. de Perrot M, Liu M, Waddell TK, Keshavjee S. Ischemia-reperfusion-induced lung injury. Am J Respir Crit Care Med. 2003;167:490-511.

28. Suzuki K. Shimazaki M, Kutsuki H. Beta2-microglobulin-selective adsorbent column (Lixelle) for the treatment of dialysis-related amyloidosis. Ther Apher Dial. 2003;7:104-7.

29. Iskender I, Sakamoto J, Nakajima D, Lin H, Chen M, Kim H, et al. Human $\alpha 1$-antitrypsin improves early post-transplant lung function: pre-clinical studies in a pig lung transplant model. J Heart Lung Transplant. 2016;35: 913-21.

30. Diamond JM, Arcasoy S, Kennedy CC, Eberlein M, Singer JP, Patterson GM, et al. Report of the International Society for Heart and Lung Transplantation working group on primary lung graft dysfunction, part II: epidemiology, risk factors, and outcomes-A 2016 consensus group statement of the International Society for Heart and Lung Transplantation. J Heart Lung Transplant. 2017;36: 1104-13

31. Gelman AE, Fisher AJ, Huang HJ, Baz MA, Shaver CM, Egan TM, et al. Report of the ISHLT working group on primary lung graft dysfunction Part III: mechanisms: A 2016 consensus group statement of the International Society for Heart and Lung Transplantation. J Heart Lung Transplant. 2017;36:1114-20.

32. Netea MG, van de Veerdonk FL, van der Meer JWM, Dinarello CA, Joosten LA. Inflammasome-independent regulation of IL-1-family cytokines. Annu Rev Immunol. 2015;33:49-77.

33. Turner MD, Nedjai B, Hurst T, Pennington DJ. Cytokines and chemokines: at the crossroads of cell signalling and inflammatory disease. Biochim Biophys Acta. $2014 ; 1843: 2563-82$

Key Words: ex vivo lung perfusion, perfusate adsoprtion, cytosorb, lung transplantation, ischemia-reperfusion injury, inflammatory response

\section{Discussion}

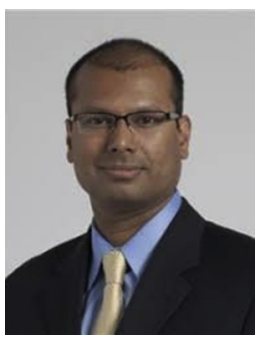

Dr Siva Raja (Cleveland, Ohio). I would like to start by commending the authors for this work but also for their prior work in this field. As EVLP is increasing, it has the potential to increase the availability of this precious resource, the organ. In this work you are describing the use of adsorbers in the EVLP circuit and showing that there is decreased inflammatory response that corresponds to an improved function. I have the following 3 questions.

As you mentioned, these are relatively nonspecific adsorption beads. How do you think this will affect future studies involving other molecules that are anti- inflammatory even though this takes up proinflammatory molecules? The reason I ask this is, one, in your own study you show that some markers such as IL-10 are actually anti-inflammatory, are also decreased, and your own prior work looking at trimetazidine, which is an anti-ischemic agent, which when used in the EVLP circuit decreases inflammation and produces the exact same result. How do you think that this technology of taking everything out will play in the role of adding things that could also decrease the effect?

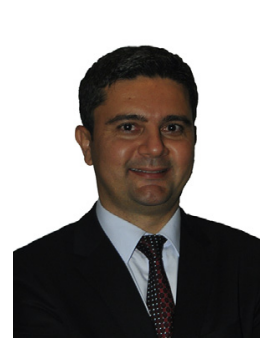

Dr Ilker Iskender (Zurich, Switzerland). I think this could be explained along with the side effects of medications. Using medications has advantages and disadvantages. How can we tackle the unwanted effects of perfusate-adsorption related to drug removal? I think one way could be administration of high-doses of medications or agents. EVLP is an isolated platform. Interventions will affect only the lungs, not all the body. One simple way is to tackle this side effect is to administration of an increased amount of therapeutics while using this adsorber in the setting of EVLP.

Dr Raja. There was an article by Kakishita and colleagues $^{18}$ in 2010 in the Annals of Thoracic Surgery that also used an adsorbent membrane in the EVLP circuit and showed that decreasing cytokines like IL-8 and tumor necrosis factor-alpha that were chemically detected to be decreased but had no physiologic effect.

So the beads that you were using, were they provided by the company or were they commercially available for other groups to test? I am trying to figure out, what's different about your beads that the last adsorption membrane was not able to show a physiologic effect?

Dr Iskender. I don't know the mechanistic difference between the 2 membranes. It's difficult for me to answer this question from that point of view at the moment. But the experimental model they used was different than this study. The EVLP was initiated after a short warm-ischemic time, whereas we have used a clinically relevant, prolonged cold ischemic injury model in this study. Together with the difference in membrane technology, the use of different experimental models may yielded discordant results between the 2 studies.

This device is available for patient use in Europe with a CE mark. The company has provided the adsorber for research purposes in this study. I think the device is currently under investigation on other parts of the world.

Dr Raja. In this study, it's a very manicured setting where the harvesting of the organ is done in a pristine fashion; there is no trauma to the lungs and so on and so forth. My interpretation of the data is that by binding or removing these inflammatory mediators and decreasing the inflammatory response, you are able to preserve better 
graft function.

But life is not so pristine. The organs are usually placed on EVLP because there is something not quite right with the organ. So how do you think these data would pan out in a setting where already the inflammatory cascade has been started? How do you think this would work in terms of reversing some of the effects? It may work in not starting it, but what are your thoughts?

Dr Iskender. True, we have tested the device in a controlled setting for research purposes, which may not simulate the real-life conditions. Nevertheless, this broadspectrum adsorbent may be advantageous, especially in the clinical setting where the donor lungs are also injured with microorganisms or aspiration, involving other endotoxins or some other harmful substances that could be further cleared with this adsorber during EVLP. This would be my speculation.

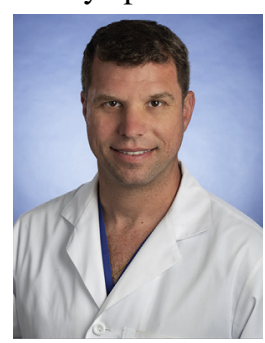

Dr Marcelo Cypel (Toronto, Ontario, Canada). Very nice work. I have 2 questions. One is, in your EVLP data in the 1-hour time point you already saw a significant difference between the 2 groups. Do you think the effect already happened early on that showed that difference at a 1-hour baseline?
I think the more interesting finding of this study to me is the postreperfusion cytokines in the lung tissue, because that really shows you did something to EVLP that decreased reperfusion injury. But is that true or was it just a matter of accumulation of those cytokines during EVLP that were different in one group than the other and just persisted in the first few hours after reperfusion or was it truly reperfusion injury? I wonder if you did, for example, percutaneous coronary intervention to look at gene expression levels rather than just protein accumulation?

Dr Iskender. To answer your first question, we started to see a physiologic improvement from the first hours of EVLP, which is actually identical to what we saw in the first article. It appears that this strategy is affecting the EVLP physiology early with the initiation of EVLP.

Regarding the second question, we have only looked at the plasma levels of cytokines, not tissue levels. All of the studied analytes were comparable except for IL1ra, which has been classified as an anti-inflammatory cytokine. It has also been secreted as an acute-phase protein from liver. We believe that elevated IL-1ra plasma levels are a surrogate marker of enhanced inflammation regulated by proinflammatory cytokines, which is in favor of the treatment effect. 


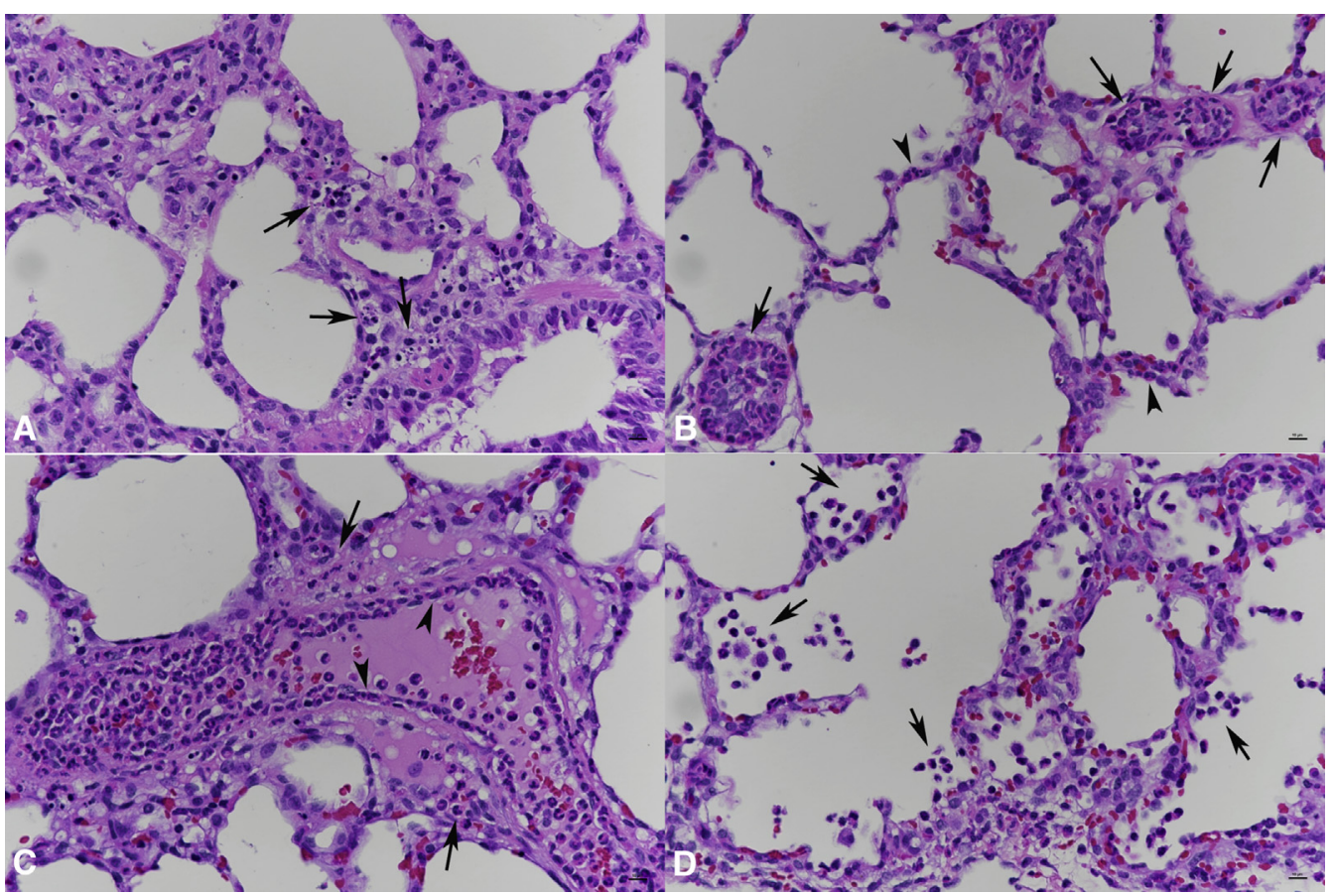

FIGURE E1. Histologic features of lungs. A, Nontransplanted right lung after EVLP with adsorber. Histologic changes are restricted to the apoptosis of leukocytes within capillaries and in the peribronchiolar tissue (arrows). B-D, Transplanted left lung from the control group at autopsy. Overall histologic score of 9. B, Most vessels (arrows) and capillaries (arrowheads) are packed with neutrophils. Score A3. C, Large vein with evidence of neutrophil emigration, represented by extensive rolling of neutrophils along endothelial cells (arrowheads) and the presence of neutrophils within the perivascular tissue (arrows). Score B3. D, Individual to groups of neutrophils are present within alveoli (arrows). Score C3. Hematoxylin-eosin stain. Bars $=10 \mu \mathrm{m}$.

TABLE E1. Quantitative presentation of the discordance between the 2 pathologists related to the microscopic lung injury at the end of transplantation

\begin{tabular}{|c|c|c|c|c|}
\hline & Score A & Score B & Score C & Score overall \\
\hline \multicolumn{5}{|c|}{ Controls } \\
\hline 1 & 1 & $1[0.5-1]$ & 1 & 3 \\
\hline 2 & $1.5[1-1.5]$ & 1 & 1 & 3.5 \\
\hline 3 & 2 & 2 & 3 & 7 \\
\hline 4 & 1 & 1 & 1.5 [1.5-2] & 3.5 \\
\hline 5 & 3 & 3 & 3 & 9 \\
\hline \multicolumn{5}{|c|}{ Adsorber } \\
\hline 1 & $2.5[\mathbf{2 - 2 . 5}]$ & 1.5 & 0 & 4 \\
\hline 2 & 1 & 2 & 1 & 4 \\
\hline 3 & 2 & 2.5 & 2 & 6.5 \\
\hline 4 & 1 & $1.5[\mathbf{1 . 5}-2]$ & 2 & 4.5 \\
\hline 5 & 1 & 1 & 0 & 2 \\
\hline
\end{tabular}

Tissue samples were stained with hematoxylin-eosin and blindly assessed by 2 veterinary pathologists for any pathological changes. The scoring system to assess lung injury. Score A: evidence of neutrophil recruitment into the lungs (1: moderate, 2: high, 3: vessels packed with neutrophils). Score B: Evidence of neutrophil emigration into the tissue (1: neutrophil rolling along vascular endothelial cells, 2: neutrophils within vessel walls, 3: neutrophils immediately outside vessels). Score C: Evidence of neutrophils within the tissue, represented by the presence of individual neutrophils within alveolar lumina (1: in rare alveoli, 2: in occasional alveoli, 3: in numerous alveoli). The results showed a concordance in most of the cases with a small disagreement in 5 tissues. Those cases showing disagreement were reevaluated with particular emphasis on the inclusion of all areas of the sections, and a final definitive score was reached. The discordant results are given in bold. 
000 Perfusate adsorption during ex vivo lung perfusion improves early posttransplant lung function

Ilker Iskender, MD, MSc, Stephan Arni, PhD, Tatsuo Maeyashiki, MD, Necati Citak, MD, Mareike Sauer, DVM, Josep Monné Rodriguez, DVM, Thomas Frauenfelder, MD, Isabelle Opitz, MD,

Walter Weder, MD, and Ilhan Inci, MD, Zurich, Switzerland

Adsorption of inflammatory mediators during EVLP improves immediate post-transplant graft function by decreasing inflammatory response to reperfusion in pigs. 